\title{
Translation of biomechanical concepts in bone tissue engineering: from animal study to revision knee arthroplasty
}

\author{
A. Roshan-Ghias ${ }^{\mathrm{a} 1}$, A. Terrier ${ }^{\mathrm{a}}$, B.M. Jolles ${ }^{\mathrm{b}}$ and D.P. Pioletti ${ }^{\mathrm{a} *}$ \\ ${ }^{a}$ Laboratory of Biomechanical Orthopedics, Ecole Polytechnique Fédérale de Lausanne, STI, IBI, Station 19, 1015 Lausanne, \\ Switzerland; ${ }^{b}$ Service of Orthopaedic Surgery and Traumatology, Centre Hospitalier Universitaire Vaudois and University of Lausanne, \\ Lausanne, Switzerland
}

(Received 1 May 2012; final version received 6 August 2012)

\begin{abstract}
Bone defects in revision knee arthroplasty are often located in load-bearing regions. The goal of this study was to determine whether a physiologic load could be used as an in situ osteogenic signal to the scaffolds filling the bone defects. In order to answer this question, we proposed a novel translation procedure having four steps: (1) determining the mechanical stimulus using finite element method, (2) designing an animal study to measure bone formation spatially and temporally using microCT imaging in the scaffold subjected to the estimated mechanical stimulus, (3) identifying bone formation parameters for the loaded and non-loaded cases appearing in a recently developed mathematical model for bone formation in the scaffold and (4) estimating the stiffness and the bone formation in the bone-scaffold construct. With this procedure, we estimated that after 3 years mechanical stimulation increases the bone volume fraction and the stiffness of scaffold by 1.5- and 2.7-fold, respectively, compared to a non-loaded situation.
\end{abstract}

Keywords: bone scaffold; total knee implant revision; mechanical loading; in vivo; finite element method; translational medicine

\section{Introduction}

During revision knee arthroplasty (RKA), some of the bone underneath the tibial tray is removed during the procedure (Dorr et al. 1986). In this situation, the surgeon normally makes a half epiphyseal cut to prepare a suitable bed for a bone substitute (Bugbee et al. 2001; Nelson et al. 2003). Currently used bone substitutes are mainly allograft (Engh 1998). A different strategy can be followed by using metallic augments (Cuckler 2004). However, these two techniques have serious drawbacks. Allografts show good osteointegration at the interface, but the bulk often has difficulties to be correctly incorporated by the host bone. Moreover, allografts always present a risk of viral infection (Parks and Engh 1997; Sculco and Choi 1998). Metallic augments do not restore bone stock and their integration with adjacent bone can be an issue (Huff and Sculco 2007; Mabry and Hanssen 2007). Thus, bone scaffold has been proposed as an alternative which could restore the bone function (Terrier et al. 2009).

Bone scaffolds used in RKA will be subjected to a direct mechanical loading from the tibial tray of the knee implant. This situation will impose to correctly design the load sharing between the implant and the scaffold in order to ensure the mechanical integrity of the knee arthroplasty procedure. However, loading can be used as an in situ osteogenic signal enhancing bone formation in the scaffold (Duty et al. 2007; Boerckel et al. 2009; Pioletti 2010). This new concept has previously been demonstrated in vivo where a controlled mechanical loading on rat knee was shown to increase the bone volume fraction (BVF) in polymeric scaffold implanted in distal femur of rat (Roshan-Ghias et al. 2010).

Although in vivo studies are central to develop new solutions in bone tissue engineering, the translation of the results obtained with animal studies to human physiology is a challenging and an imperfect technique (Muschler et al. 2010). The size and shape of typical bone defects in humans are rather different from bone defect models in animals. The obtained results on the bone formation in the scaffold when mechanical loading is applied (RoshanGhias et al. 2010) can then not be directly used to design a similar strategy in RKA.

To translate animal study results into information usable for clinical applications, a theoretical model of bone formation in scaffold should be employed. We have previously developed and validated a mathematical model of bone formation in the scaffold by considering that this process follows a diffusion phenomenon (Roshan-Ghias, Vogel, et al. 2011). The model with identified parameters successfully predicted the results of two different animal experiments. In parallel, we have quantified in an animal study the bone formation inside the bone scaffold in loaded and non-loaded conditions (Roshan-Ghias, Lambers, et al. 2011). The goal of this study was to determine whether a

\footnotetext{
*Corresponding author. Email: dominique.pioletti@epfl.ch 
physiologic load transmitted by the tibial tray of the knee implant to the underlying scaffold can be used as an in situ osteogenic signal. An increase of the bone formation and mechanical property of the loaded scaffold should then be obtained compared to a situation with no mechanical loading.

\section{Materials and methods}

A schematic representation of the proposed method is shown in Figure 1. The schema has four boxes representing the four main steps followed in the study. The result of each box is the input of the next box. The process begins with a definition of the clinical application (Box 1). In our case, we wanted to know whether loading could be an osteogenic factor in a scaffold used for a RKA scaffold. To determine what mechanical stimulus is induced in the scaffold due to the physiologic loading, we devise a finite element study. By finding the mechanical stimulus, we design an animal study (Box 2) in which the loading condition in the animal is such that the level of mechanical stimulus in the scaffold is similar to that of the targeted clinical application. Bone formation over time inside the scaffold is obtained by longitudinal micro-CT imaging (Roshan-Ghias, Lambers, et al. 2011). In addition, micro-CT imaging provides spatial information on the bone formation in the scaffold. Spatial and temporal experimental data for loaded and non-loaded scaffolds are then inputted into the bone formation model developed previously (Roshan-Ghias, Vogel, et al. 2011), and the corresponding sets of parameters (one for loaded and another for non-loaded scaffolds) of the model are identified accordingly (Box 3). The bone formation model with the two sets of identified parameters allows comparing bone formation in RKA scaffold with or without mechanical stimulation. In order to estimate the mechanical properties of the RKA scaffolds, a relation between the bone volume and Young's modulus has to be established. Micro-FE modelling is used to determine this relation. The final step is to estimate the stiffness of the RKA scaffold (Box 4).

\subsection{Defining clinical application: finite element model of RKA (Box 1)}

A cadaveric tibia was scanned using a medical CT scanner (LightSpeed Ultra, General Electrics, Hertfordshire, UK). The cortical bone and trabecular bone were segmented using Amira (Visual Imaging, San Diego, CA, USA), and were prepared for meshing by Geomagic (Durham, NC, USA). The model was then imported in ABAQUS (Simulia, Providence, RI, USA) and meshed with tetrahedral elements.

The RKA prosthesis was a revision knee prosthesis prototype, which is derived from the FIRST prosthesis (Symbios Orthopédie SA, Yverdon, Switzerland). The prosthesis was composed of three parts: the stem, the plate and the polyethylene (PE). The implant geometry was simplified by removing the unnecessary edges and grooves in SolidWorks (Dassault Systèmes SolidWorks, Concorde, MA, USA) and imported in ABAQUS. The implant was placed inside the tibia under the supervision of a senior surgeon. In order to simulate the reaming process, the implant stem was enlarged by $1 \mathrm{~mm}$ and its intersection with bone was removed. The space between stem and bone was filled with cement elements (Figure 2(a)). The mechanical properties of all parts are listed in Table 1. Young's modulus of scaffold was measured in compression test using an

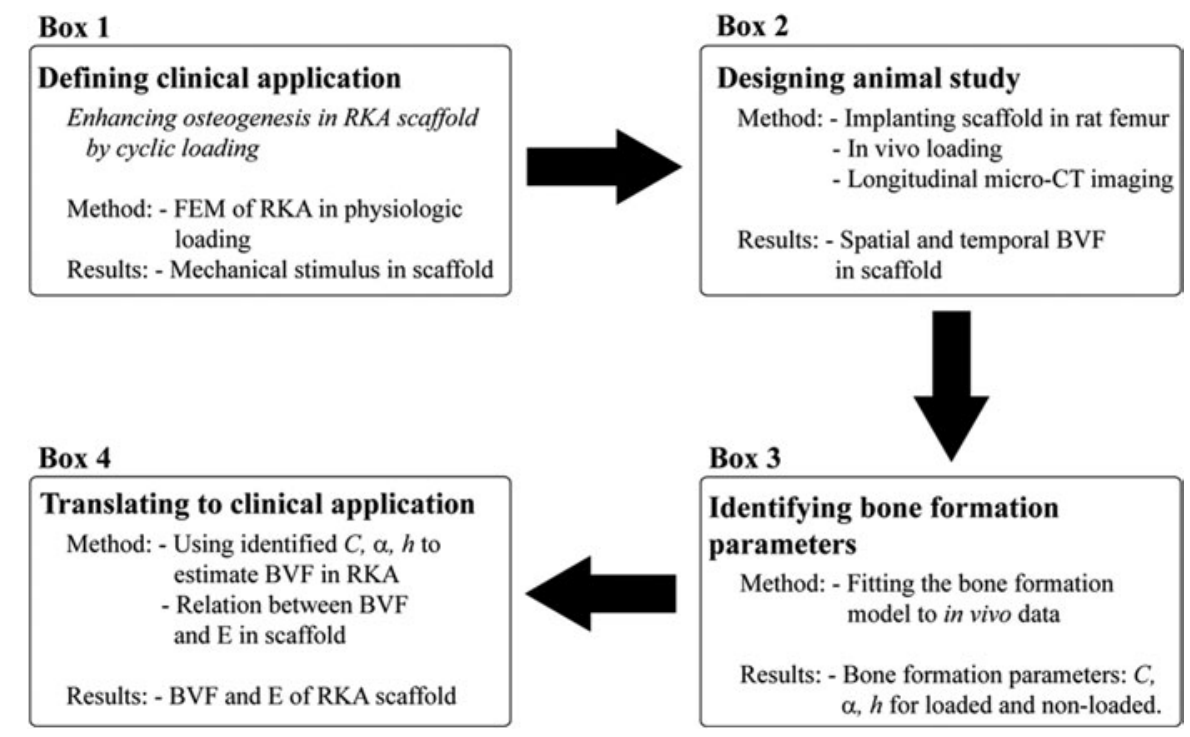

Figure 1. Schematic diagram of the proposed method, from animal study to clinical application. 

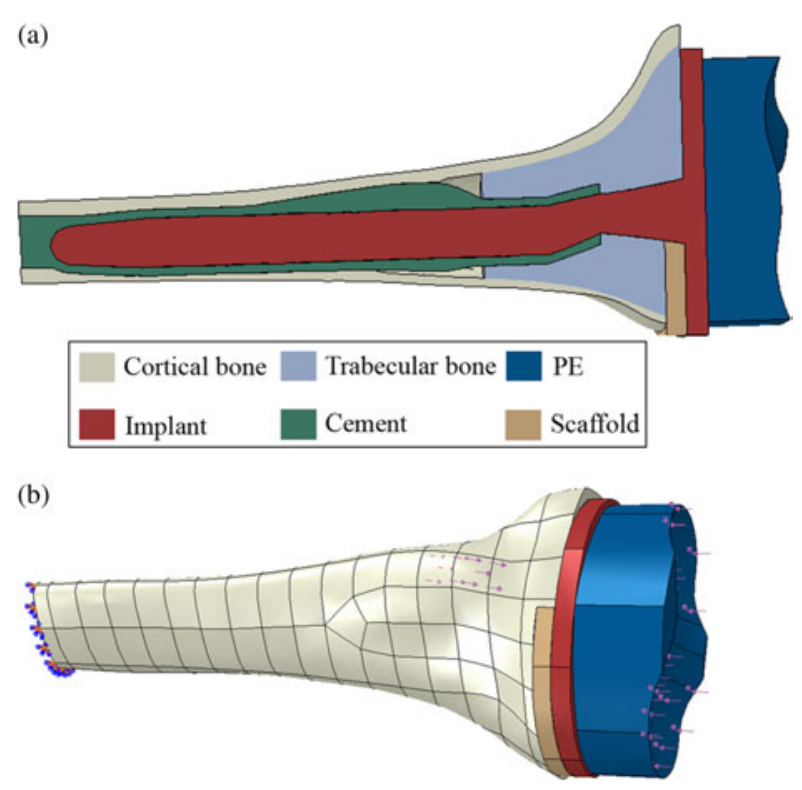

Figure 2. (a) A frontal cross section of FE model. (b) Boundary condition at the distal side of tibia. Loads due to walking are shown with arrows.

Instron Electropuls E3000 (Instron, Bucks, UK). Mechanical properties of prosthesis parts were obtained from the manufacturer.

As boundary conditions, the distal part of the bone and cement was fully constrained (Figure 2(b)). The loads were applied based on an average body weight (BW) of $86 \mathrm{~kg}$ of 62 patients who underwent RKA (Hockman et al. 2005). Assuming level walking, axial force of $2.5 \mathrm{BW}$ was applied on the PE (60\% medial, $40 \%$ lateral), shear force of $0.5 \mathrm{BW}$ was applied on the PE-directed anteriorly and patello-femoral force of $0.8 \mathrm{BW}$ was applied to the insertion point of the patellar ligament on the tibia (Figure 2(b) (Taylor et al. 1998). The contact between all parts was assumed to be glued (Completo et al. 2008). The outcome of the RKA model was the average value of strain induced in the RKA scaffold.

Mechanical stimulus for bone remodelling is defined as (Turner 1998)

$$
S=k \varepsilon f,
$$

where $S$ is the mechanical stimulus, $\varepsilon$ is the peak-to-peak strain magnitude, $f$ is the loading frequency in cycles per second and $k$ is a constant unitless coefficient. Assuming walking load $(f=1), k=1$, and having $\varepsilon$ calculated from the FEM, $S$ can be estimated.

\subsection{Designing animal study (Box 2)}

The animal study was previously performed and reported thoroughly elsewhere (Roshan-Ghias, Lambers, et al. 
2011). In short, scaffolds made of PLA/ $\beta$-TCP were inserted in both femoral condyles of rat. The right leg of each animal was subjected to loading starting from 2 weeks after surgery $(10 \mathrm{~N}, 4 \mathrm{~Hz}, 5 \mathrm{~min}$, every 2 days for a total of five times) and the left leg was kept as control. The magnitude and frequency of loading were determined such that the mechanical stimulus, $S$ (Equation (1)), in the implanted scaffold is close to the calculated mechanical stimulus in RKA scaffold obtained from Box 1 (RoshanGhias et al. 2010). The BVF in the animal study was measured using micro-CT imaging at different time points and into 16 donut-shaped concentric regions in the scaffold (Roshan-Ghias, Vogel, et al. 2011). Spatial and temporal BVF data were then obtained for loaded and control scaffolds.

\subsection{Identifying bone formation parameters (Box 3)}

We have previously shown that bone formation inside a scaffold can be described by diffusion Equation (RoshanGhias, Vogel, et al. 2011)

$$
\frac{\partial c}{\partial t}=\alpha \Delta c
$$

where $c$ is the BVF, $t$ is the time and $\alpha$ is the scaffold osteoconductivity. The basic assumption of the proposed model was that bone initiates from the surrounding osteogenic tissue (e.g. bone or bone marrow), and not from the non-osteogenic neighbours (e.g. implant and muscle). This is translated into the following boundary conditions

$$
-\left.\alpha \frac{\partial c}{\partial X}\right|_{X=\Omega_{1}}=h[C-c(X, t)]_{X=\Omega_{1}},\left.\quad \frac{\partial c}{\partial X}\right|_{X=\Omega_{2}}=0
$$

where $h$ is the peri-scaffold osteoinductivity, $C$ is the final BVF, $X$ is the spatial coordinate and $\left(\Omega_{1}\right.$ and $\Omega_{2}$ are the coordinates of the osteogenic and non-osteogenic interfaces, respectively. To identify $C, \alpha$ and $h$ for the loaded and non-loaded scaffolds, we used spatial and temporal evolution of BVF from the corresponding data obtained from the animal study described in Box 2 (Roshan-Ghias, Lambers, et al. 2011).

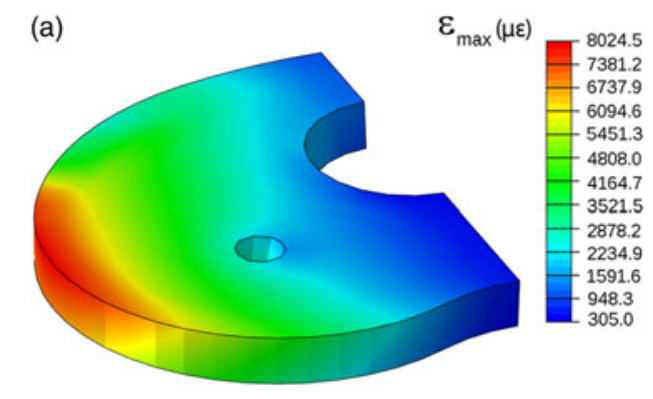

\subsection{Translating to clinical application: RKA scaffold (Box 4)}

The geometry of scaffolds used in the FEM of RKA was imported in COMSOL (Comsol AB, Stockholm, Sweden), and meshed with tetrahedral elements. Two different sets of identified parameters for the bone formation model were used (obtained from Box 3) for non-loaded and loaded scaffolds. Diffusion equation was solved inside the scaffold, assuming that the boundaries adjacent to the bone are osteogenic (Roshan-Ghias, Vogel, et al. 2011), and the boundaries adjacent to implant and muscle tissue are nonosteogenic. The two models (non-loaded and loaded) were solved for a time period of 36 months, with time steps of 1 month. In order to estimate the stiffness of RKA scaffold over time, we estimated the relation between BVF and Young's modulus of bone-scaffold construct $E(\mathrm{BVF})$ (Appendix). The BVF values from the diffusion problem and the $E(\mathrm{BVF})$ relation were used to define Young's modulus of each element. Poisson's ratio was assumed to be 0.3 for all elements. The distal part of the scaffold was fixed and the proximal part was subjected to displacement, and the apparent Young's modulus of RKA scaffold was calculated.

\section{Results}

\subsection{Box 1}

Figure 3(a) shows the distribution of the largest principal strain inside a RKA scaffold. The strain increased from around 300 to $8000 \mu \varepsilon$ in the lateral-medial direction. The average strain inside the scaffold was $3100 \mu \varepsilon$. Thus, using Equation (1), the average mechanical stimulus in RKA scaffold was equal to $3100 \mu \varepsilon / \mathrm{s}$.

\subsection{Boxes 2 and 3}

Results of the animal study with mechanical stimulus corresponding to that determined in Box 1 have been presented in Roshan-Ghias, Lambers, et al. (2011). Briefly, the mechanical stimulation enhanced the bone formation inside the scaffold by $18 \%$ after 35 weeks. The parameters of the mathematical model for the loaded and non-loaded

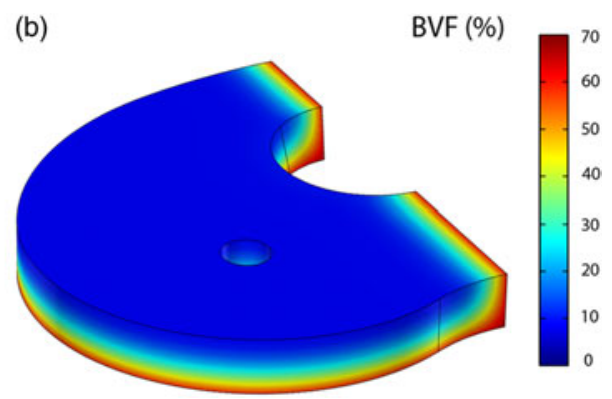

Figure 3. (a) Largest principal strain due to a load corresponding to walking. (b) BVF in a loaded scaffold after 3 years. 
scaffold are reported in Table 2. The estimated final BVF, $C$, was increased in the scaffold by mechanical stimulation. Scaffold osteoconductivity, $\alpha$, was also higher for the loaded scaffold by more than twofold, but the peri-scaffold osteoinductivity, $h$, was lower for the loaded scaffold.

\subsection{Box 4}

Figure 3(b) shows the BVF in a 3-year time for a loaded scaffold. We observed a sharp gradient of bone formation. The regions close to the bone interface reached the highest values of BVF, but the regions far from the bone interface stayed almost empty (around $10-15 \%$ of BVF). The evolution of BVF over time is shown in Figure 4(a) for both loaded and non-loaded scaffolds. Without the loading, BVF reached $28 \%$ after 3 years. However, with the loading, we observed a 1.5 -fold increase up to $42 \%$ of BVF in scaffold.

The relation between Young's modulus of bone-scaffold constructs and BVF is shown in Figure 5. We observed that as soon as one layer of bone is formed around the pores of the scaffold (equal to 15-20\% of BVF), Young's modulus of the bone-scaffold construct jumps to higher values (from 20 to 250-350 MPa). Power law was used to fit these data, as an explicit form was needed later in the RKA model:

$$
E(\mathrm{BVF})=24.2+5.65(\mathrm{BVF})^{1.457}
$$

where $E$, Young's modulus, is expressed in $\mathrm{MPa}$.

By using Equation (2) and the estimated BVF inside RKA scaffold, the stiffness of the RKA scaffold was estimated over time. It showed a different temporal pattern compared to BVF (Figure 4(b)). In the first 10-12 months for the non-loaded

Table 2. Bone formation parameters for non-loaded and loaded scaffolds.

\begin{tabular}{lcc}
\hline & Non-loaded & Loaded \\
\hline$C(\%)$ & 68.6 & 83.5 \\
$\alpha\left(\mathrm{mm}^{2} /\right.$ day $)$ & 0.0041 & 0.0097 \\
$H(\mathrm{~mm} /$ day $)$ & 0.0073 & 0.0057 \\
\hline
\end{tabular}

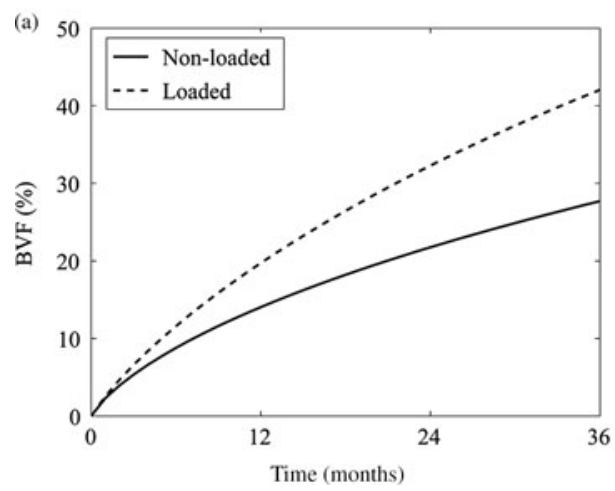

scaffold, and the first 4-5 months for the loaded scaffold, a latency period was observed where Young's modulus of the bone-scaffold construct does not markedly increase over time. The stiffness of the non-loaded scaffold reached $550 \mathrm{MPa}$, whereas for the loaded scaffold it reached $1500 \mathrm{MPa}$. It was also observed that the loaded scaffold reached the stiffness of $550 \mathrm{MPa}$ as early as 15 months, compared to 36 months needed for the non-loaded scaffold.

\section{Discussion}

The goal of this study was to determine whether a physiologic load transmitted by the tibial tray of the RKA to the underlying scaffold could be used as an in situ osteogenic signal. We proposed a novel procedure to answer this question. The procedure contains finite element modelling, animal experiment, identification of bone formation and translation of the animal study to the clinical application. We found out that the mechanical stimulation transmitted by a tibial tray of a RKA is indeed a potent osteogenic signal, enhancing the bone formation by $50 \%$ and the stiffness of bone scaffold by 2.7 -fold.

The FE model of RKA allowed us to test whether the scaffold can withstand the loading due to walking. Various parameters might affect the outcome of the model, and we have addressed some of these issues in a previous publication (Terrier et al. 2009). The highest strain induced inside the scaffold occurred at the most superficial part of the scaffold, but was well below $1 \%$ strain. It is within the safe values of strain (Terrier et al. 2009). The same FE model was used to estimate the mechanical stimulus in the scaffold. The average mechanical stimulus in the RKA scaffold was equal to $3100 \mu \varepsilon / \mathrm{s}$. In the animal experiment, we have shown that the average of the largest principal strain in the scaffold was $625 \mu \varepsilon$ (Roshan-Ghias et al. 2010). As the frequency of loading was $4 \mathrm{~Hz}$, the mechanical stimulus, $S$, is estimated to be equal to $2500 \mu \varepsilon / \mathrm{s}$, close to the RKA value. The new concept of using controlled mechanical stimulus applied on the scaffold has also been recently suggested for other surgical procedures (Pioletti 2010).

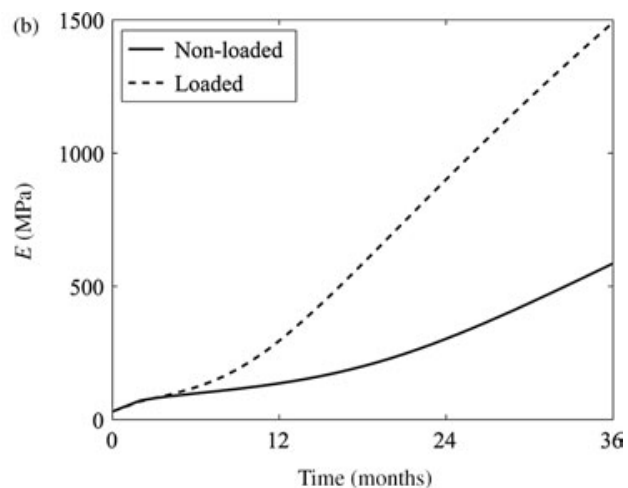

Figure 4. Evolution of (a) BVF. (b) Young's modulus, over time for non-loaded and loaded scaffolds. 


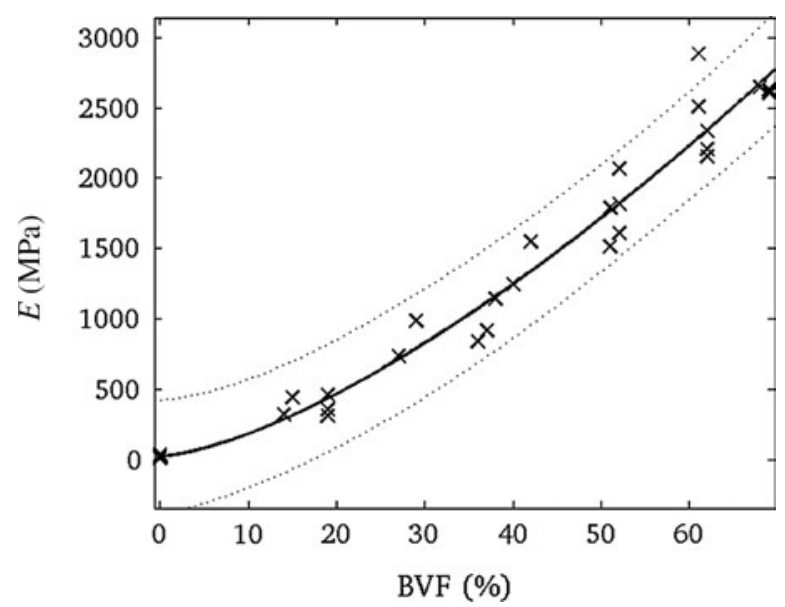

Figure 5. Young's modulus of a unit of bone-scaffold construct with respect to BVF. The cross points are the estimated values for each scaffold, the solid line is the fitted relation and the dotted lines are the $95 \%$ confidence interval of the fitted relation.

Without mechanical stimulation, the calculations indicated that the scaffold would be poorly filled $(27 \%)$ with bone after 3 years. However, in the presence of mechanical stimulation, the BVF was estimated to reach $43 \%$. This would be a remarkable increase without adding extra cells and growth factors. Moreover, the mechanical properties increased by 2.7 -fold, reaching almost $1.5 \mathrm{GPa}$, similar to the trabecular bone of human proximal tibia (Guo 2001). However, the interface between scaffold and implant showed little bone formation (10-20\%), even after 3 years. Thus, if a faster regeneration of bone is needed, other means of osteoinduction should be employed. Cell seeding (Puelacher et al. 1996; Vacanti et al. 2001; Pioletti et al. 2006; Degano et al. 2008) and growth factor (Zisch et al. 2003; Seliktar et al. 2004) impregnation of scaffold enhance the rate of bone formation by a great extent, and are possible solutions to accelerate the bone formation inside the scaffold. However, as mentioned in the introduction, this comes with an increased complexity, which may hamper clinical translation of bone tissue engineering.

The findings of this study suggest that early mechanical stimulation can be highly beneficial for the bone formation in the scaffold. The current practice, however, is to immobilise the knee in a cast for at least 3 months, and full weight bearing is inhibited until a maximum of 3 months post-operatively (Heyligers et al. 2001). Indeed, post-operative load bearing has always been a disputable topic in orthopaedics (Ghazavi et al. 1997). It has also been suggested that the limb could be immediately loaded with half $\mathrm{BW}$, if the joint is perceived to be stable (Pietsch et al. 2006). Late loading is not as osteogenic as early loading, as it has been shown before in fracture healing (Goodship et al. 1998). Thus, based on the results of this study, we could propose to apply short bouts of daily loading (normal walking load) in the first month post-surgery, with initial delay of 1 week (Roshan-Ghias, Lambers, et al. 2011). The duration of loading can be as short as half an hour. We applied cyclic loading at $4 \mathrm{~Hz}$ for $5 \mathrm{~min}$, so in order to have the same number of cycles of loading during walking, 20-30 min can be sufficient. However, this can be recommended if surgeon perceived that the joint is stable and capable of supporting load.

In the absence of clinical data for the bone formation inside the scaffold, we had no choice but to use the animal study data. Moreover, the in vivo data that we had were from a small animal like rat. It is generally suggested that larger animals such as goat and sheep are more suitable for bone-related studies, as they show closer resemblance to human bone (Pearce et al. 2007). However, some researchers have reported that the rate of bone ingrowth into a bone chamber implanted in rat and goat is similar (van der Donk et al. 2001; Buma et al. 2004). This might suggest that the rate of bone formation is the same for human as well. However, age plays an important role in human on the healing capacity of the bone (Gruber et al. 2006). Since the patients who undergo RKA normally belong to an old population, the rate of bone formation might be significantly lower. If the surrounding bone is sclerotic and poorly vascularised, it may not allow for incorporation of bone substitute (Nelson et al. 2003).

One of the limitations of our model is that we extrapolated the data in time. The in vivo data we had were limited to 8 months, while we extrapolated it to 3 years time. The scaffold can degrade to a great extent after 3 years, and this might affect the process of bone formation. Degradation could also influence the mechanical properties of the bone-scaffold construct over time. However, the effect of degradation on mechanical properties will have a limited impact, as bone supports most of the load in a bone-scaffold construct due to its superior mechanical properties compared to scaffold. We did not take into account the effect of degradation in this study. Another possible limitation of the model to evaluate the bone formation in the scaffold could be that the solution of diffusion equation at $t=0$ might be erroneous in some cases. However, as we were dealing with bone formation as early as 2 weeks, and not at $t=0$, we believe (and the result shows) that the mentioned drawback of Fick's Law does not affect our result.

One of our assumptions was that Young's modulus of the bone-scaffold construct mainly depends on the BVF of scaffold. We have shown that BVF alone cannot explain all the variations seen between Young's modulus of bonescaffold constructs; however, it is by far the most important bone morphometric parameter in determining stiffness (Roshan-Ghias, Lambers, et al. 2011). We also assumed that bone is formed in layers inside the scaffold, but in reality bone is formed in patches, as seen at the early time points using micro-CT (Roshan-Ghias, Vogel, et al. 2011). However, as soon as BVF is large enough, the 
patches grow and join to make a unified layer around the walls of the pores (observed in histology sections, in Roshan-Ghias, Lambers, et al. (2011)).

\section{Conclusions}

In this study, we proposed that mechanical stimulation could be used as an efficient osteogenic signal for bone formation in a scaffold filling an epiphyseal defect in RKA. Our results suggested that loading could enhance BVF and stiffness of the scaffold. We proposed a new procedure to translate animal study data into a clinical application. The proposed method can be employed for evaluation of other bone tissue engineering strategies for different clinical applications.

\section{Acknowledgements}

This project was supported by a SNSF grant (No. 205320121893) and the Inter-Institutional Center for Translational Biomechanics EPFL-CHUV-DAL.

\section{Note}

1. Current address: Laboratory for Stem Cells and Tissue Engineering, Department of Biomedical Engineering, Columbia University, New York, USA.

\section{References}

Boerckel JD, Dupont KM, Kolambkar YM, Lin AS, Guldberg RE. 2009. In vivo model for evaluating the effects of mechanical stimulation on tissue-engineered bone repair. $\mathrm{J}$ Biomech Eng. 131(8):084502.

Bugbee WD, Ammeen DJ, Engh GA. 2001. Does implant selection affect outcome of revision knee arthroplasty? J Arthroplasty. 16(5):581-585.

Buma P, Schreurs W, Verdonschot N. 2004. Skeletal tissue engineering-from in vitro studies to large animal models. Biomaterials. 25(9):1487-1495.

Completo A, Simoes JA, Fonseca F, Oliveira M. 2008. The influence of different tibial stem designs in load sharing and stability at the cement-bone interface in revision TKA. Knee. 15(3):227-232.

Cuckler JM. 2004. Bone loss in total knee arthroplasty: graft augment and options. J Arthroplasty. 19(4 Suppl. 1):56-58.

Degano IR, Vilalta M, Bago JR, Matthies AM, Hubbell JA, Dimitriou H, Bianco P, Rubio N, Blanco J. 2008. Bioluminescence imaging of calvarial bone repair using bone marrow and adipose tissue-derived mesenchymal stem cells. Biomaterials. 29(4):427-437.

Dorr LD, Ranawat CS, Sculco TA, McKaskill B, Orisek BS. 1986. Bone graft for tibial defects in total knee arthroplasty. Clin Orthop Relat Res. 205:153-165.

Duty AO, Oest ME, Guldberg RE. 2007. Cyclic mechanical compression increases mineralization of cell-seeded polymer scaffolds in vivo. J Biomech Eng. 129(4):531-539.

Engh GA. 1998. The use of bulk allografts in revision knee arthroplasty. Oper Techniq Orth. 8(3):153-157.

Ghazavi MT, Stockley I, Yee G, Davis A, Gross AE. 1997. Reconstruction of massive bone defects with allograft in revision total knee arthroplasty. J Bone Joint Surg Am. 79(1): 17-25.

Goodship AE, Cunningham JL, Kenwright J. 1998. Strain rate and timing of stimulation in mechanical modulation of fracture healing. Clin Orthop Relat Res. 355:S105-S115.

Gruber R, Koch H, Doll BA, Tegtmeier F, Einhorn TA, Hollinger JO. 2006. Fracture healing in the elderly patient. Exp Gerontol. 41(11):1080-1093.

Guo XE. 2001. Mechanical properties of cortical bone and cancellous bone tissue. In: Cowin S, editor. Bone mechanics handbook. Boca Raton: CRC.

Heyligers IC, van Haaren EH, Wuisman PI. 2001. Revision knee arthroplasty using impaction grafting and primary implants. J Arthroplasty. 16(4):533-537.

Hockman DE, Ammeen D, Engh GA. 2005. Augments and allografts in revision total knee arthroplasty: usage and outcome using one modular revision prosthesis. J Arthroplasty. 20(1):35-41.

Homminga J, Huiskes R, Van Rietbergen B, Ruegsegger P, Weinans H. 2001. Introduction and evaluation of a grayvalue voxel conversion technique. J Biomech. 34(4):513-517.

Huff TW, Sculco TP. 2007. Management of bone loss in revision total knee arthroplasty. J Arthroplasty. 22(7):32-36.

Mabry TM, Hanssen AD. 2007. The role of stems and augments for bone loss in revision knee arthroplasty. J Arthroplasty. 22(4 Suppl. 1):56-60.

Muschler GF, Raut VP, Patterson TE, Wenke JC, Hollinger JO. 2010. The design and use of animal models for translational research in bone tissue engineering and regenerative medicine. Tissue Eng Part B Rev. 16(1):123-145.

Nelson CL, Lonner JH, Rand JA, Lotke PA. 2003. Strategies of stem fixation and the role of supplemental bonegraft in revision total knee arthroplasty. J Bone Joint Surg Am. 85:A52-A57.

Parks NL, Engh GA. 1997. The Ranawat Award. Histology of nine structural bone grafts used in total knee arthroplasty. Clin Orthop Relat Res. 345:17-23.

Pearce AI, Richards RG, Milz S, Schneider E, Pearce SG. 2007. Animal models for implant biomaterial research in bone: a review. Eur Cell Mater. 13:1-10.

Perren SM. 1979. Physical and biological aspects of fracture healing with special reference to internal fixation. Clin Orthop Relat Res. 138:175-196.

Pietsch M, Hofmann S, Wenisch C. 2006. Treatment of deep infection of total knee arthroplasty using a two-stage procedure. Oper Orthop Traumatol. 18(1):66-87.

Pioletti DP, Montjovent MO, Zambelli PY, Applegate L. 2006. Bone tissue engineering using foetal cell therapy. Swiss Med Wkly. 136(35-36):557-560.

Pioletti DP. 2010. Biomechanics in bone tissue engineering. Comput Meth Biomech Biomed Eng. 13(6):837-846.

Puelacher WC, Vacanti JP, Ferraro NF, Schloo B, Vacanti CA. 1996. Femoral shaft reconstruction using tissue-engineered growth of bone. Int J Oral Maxil Surg. 25(3):223-228.

Rho JY, Hobatho MC, Ashman RB. 1995. Relations of mechanical properties to density and CT numbers in human bone. Med Eng Phys. 17(5):347-355.

Roshan-Ghias A, Terrier A, Bourban PE, Pioletti DP. 2010. In vivo cyclic loading as a potent stimulatory signal for bone formation inside tissue engineering scaffold. Eur Cell Mater. 19:41-49.

Roshan-Ghias A, Lambers F, Gholam-Rezaee M, Müller R, Pioletti DP. 2011. In vivo loading increases mechanical 
properties of scaffold by affecting bone formation and bone resorption rate. Bone, 49(10):1357-1364.

Roshan-Ghias A, Vogel A, Rakotomanana L, Pioletti DP. 2011. Prediction of spatio-temporal bone formation in scaffold by diffusion equation. Biomaterials. 32(29):7006-7012.

Sculco TP, Choi JC. 1998. The role and results of bone grafting in revision total knee replacement. Orthop Clin North Am. 29(2):339-346.

Seliktar D, Zisch AH, Lutolf MP, Wrana JL, Hubbell JA. 2004. MMP-2 sensitive, VEGF-bearing bioactive hydrogels for promotion of vascular healing. J Biomed Mater Res A. 68(4):704-716.

Taylor SJ, Walker PS, Perry JS, Cannon SR, Woledge R. 1998. The forces in the distal femur and the knee during walking and other activities measured by telemetry. J Arthroplasty. 13(4):428-437.

Terrier A, Sedighi-Gilani M, Roshan Ghias A, Aschwanden L, Pioletti DP. 2009. Biomechanical evaluation of porous biodegradable scaffolds for revision knee arthroplasty. Comput Meth Biomech Biomed Eng. 12(3):333-339.

\section{Appendix: Relation between $\mathrm{BVF}$ and $\mathrm{E}$ in the scaffold}

Five cubic scaffolds were scanned by micro-CT and the images were reconstructed with NRecon software (SkyScan, Kontich, Belgium). The obtained images were then imported in Matlab (Mathworks, Natick, MA, USA) to segment scaffold from pores and produce voxel-based micro-FE models (Figure 6). The region of interest was selected as a cube of $2.5 \mathrm{~mm}$ in length. PLA material properties were estimated using back-calculation technique (Van Rietbergen et al. 1997), i.e. by comparing micro-FE analysis and experimental compression test performed on the scaffold. To model the bone formation in the scaffold, it
Turner CH. 1998. Three rules for bone adaptation to mechanical stimuli. Bone. 23(5):399-407.

Vacanti CA, Bonassar LJ, Vacanti MP, Shufflebarger J. 2001. Replacement of an avulsed phalanx with tissue-engineered bone. New Eng J Med. 344(20):1511-1514.

van der Donk S, Buma P, Aspenberg P, Schreurs BW. 2001. Similarity of bone ingrowth in rats and goats: a bone chamber study. Comp Med. 51(4):336-340.

Van Rietbergen B, Kabel J, Odgaard A, Huiskes R. 1997. Determination of trabecular bone tissue elastic properties by comparison of experimental and finite element results. In: Sol H. Oomens C.W.J, editors. Material identification using mixed numerical experimental methods. Netherlands: Kluwer Academic Publishers, p. 183-192.

Webb JC, Spencer RF. 2007. The role of polymethylmethacrylate bone cement in modern orthopaedic surgery. J Bone Joint Surg Br. 89(7):851-857.

Zisch AH, Lutolf MP, Hubbell JA. 2003. Biopolymeric delivery matrices for angiogenic growth factors. Cardiovasc Path. 12(6):295-310.

was assumed that bone was uniformly opposed layer-by-layer on the scaffold. Each layer is represented by one element in the micro-FE model. To simulate different BVFs in the scaffold, one to five layers of elements were created on the walls of the scaffold (Figure 6). The remaining spaces in pores were assumed to be filled with elements representing bone marrow. The model was then exported to ABAQUS for structural analysis. Mechanical properties are shown in Table 3. Strain of $2.5 \%$ was applied to the bone-scaffold constructs and Young's modulus was calculated accordingly. A mathematical relation, $E(\mathrm{BVF})$, between $\mathrm{BVF}$ and Young's modulus could then be established.

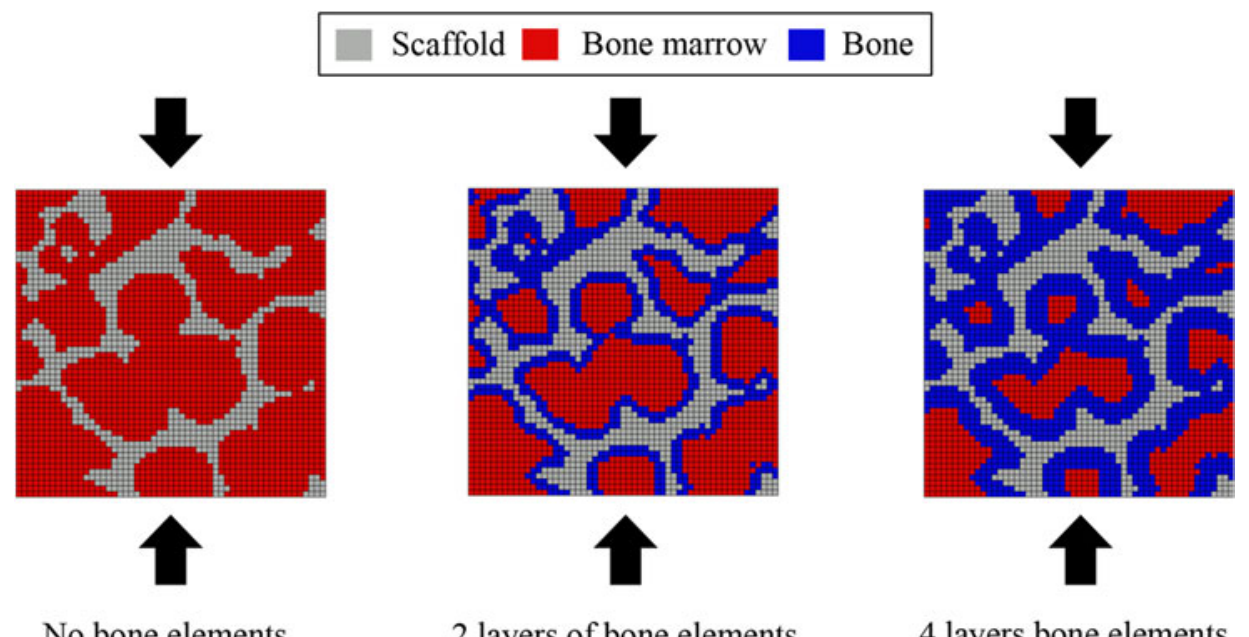

Figure 6. A cross section of the FE mesh of bone-scaffold construct, showing growth of bone elements on the pore walls. The arrows show the direction of the load.

Table 3. Mechanical properties of materials used in micro-FE model.

\begin{tabular}{lcc}
\hline & $E(\mathrm{MPa})$ & $v$ \\
\hline PLA & 313 & 0.3 \\
Bone (Homminga et al. 2001) & 5000 & 0.3 \\
Bone marrow (Perren 1979) & 0.05 & 0.3 \\
\hline
\end{tabular}

